\title{
Participación digital del público en la ciencia de excelencia española: análisis de los proyectos financiados por el European Research Council
}

\author{
Digital public engagement in excellent Spanish \\ science: Analysis of research projects funded by \\ European Research Council
}

\author{
Lourdes López-Pérez; María-Dolores Olvera-Lobo
}

Cómo citar este artículo:

López-Pérez, Lourdes; Olvera-Lobo, María-Dolores (2019). “Participación digital del público en la ciencia de excelencia española: análisis de los proyectos financiados por el European Research Council". El profesional de la información, v. 28, n. 1, e280106.

https://doi.org//10.3145/epi.2019.ene.06

Artículo recibido el 17-08-2018

Aceptación definitiva: 04-12-2018

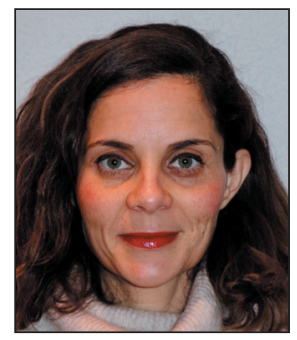

Lourdes López-Pérez $\square$

https://orcid.org/0000-0002-5787-1531

Universidad de Granada

Facultad de Comunicación y Documentación

Colegio Máximo de Cartuja.

Campus Universitario de Cartuja.

18071 Granada, España

lourdes.Ipez@gmail.com

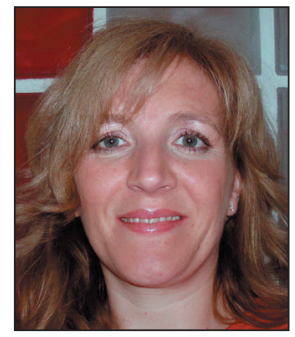

María-Dolores Olvera-Lobo

https://orcid.org/0000-0002-0489-7674

Universidad de Granada

Facultad de Comunicación y Documentación

Colegio Máximo de Cartuja.

Campus Universitario de Cartuja.

18071 Granada, España

molvera@ugr.es

\section{Resumen}

Se analiza cómo la ciencia excelente usa las aplicaciones web 2.0 para fomentar la participación del público en el proceso de investigación. Se entiende participación desde la perspectiva de la investigación e innovación responsables, no sólo como la comunicación de los resultados científicos, sino también como la implicación de la sociedad en el desarrollo científico. Para el análisis se ha diseñado y validado una encuesta ad hoc mediante el método Delphi. La muestra de estudio está constituida por los proyectos españoles financiados por el European Research Council en 2015. Entre los resultados obtenidos destaca el reducido uso de las herramientas digitales. De hecho, sólo el 23,9\% de los proyectos tiene web y menos de un 15\% perfiles sociales. En cuanto a los mecanismos de participación, un limitado 3\% utiliza la web 2.0 para implicar a los ciudadanos. Sobre las características de la comunicación se puede concluir que es unidireccional desde la institución-responsable del proyecto hacia la sociedad -97\% de los casos-y presenta un alto nivel de especialización -en más del $80 \%$ de los casos se utiliza un lenguaje técnico y los mensajes publicados están dirigidos a expertos-.

\section{Palabras clave}

Comunicación científica; Comunicación pública de la ciencia; Participación; Medios sociales; Redes sociales; Internet; Investigación e innovación responsables; RRI; Proyectos de investigación; Método Delphi.

\begin{abstract}
How much the excellent science uses web 2.0 tools to encourage public engagement in the research process is analyzed. Understanding participation, from the perspective of responsible research and innovation, not only as the communication of scientific results, but also the involvement of society in scientific development. For the analysis, an ad-hoc questionnaire developed using the Delphi method was designed and validated. The study sample is constituted by Spanish projects financed by the European Research Council in 2015. Among the results obtained, the reduced use of digital applications stands out. In fact, only $23.9 \%$ of projects have a website and less than $15 \%$ have a social profile. Regarding participation mechanisms, a limited $3 \%$ uses web 2.0 to involve citizens. With reference to the characteristics of the
\end{abstract}


communication, it can be concluded that it is unidirectional from the institution responsible for the project to society $-97 \%$ of the cases- and presents a high level of specialization -in more than $80 \%$ of the cases a technical language is used and the messages posted are aimed solely at experts-.

\section{Keywords}

Science communication; Public communication in science; Participation; Social networks; Social media; Internet; Responsible research and innovation; RRI; Research projects; Delphi method.

\section{Introducción}

La transformación de la relación entre ciencia y sociedad se ha reflejado en la bibliografía académica como un cambio de objeto de estudio, influido por el acercamiento de ambas en las dos últimas décadas. Se ha trasladado el foco de interés de la alfabetización científica del público a su implicación en el proceso científico; de la comunicación al diálogo; de la mera unión de ciencia y sociedad a una ciencia hecha con y para la sociedad; y del modelo de déficit cognitivo a la implicación de la sociedad en el proceso científico (Marschalek, 2017). Así hasta llegar al actual modelo de investigación e innovación responsable propiciado por la Comisión Europea (2014), que defiende que los procesos y los resultados científicos estén alineados con los valores, necesidades y expectativas de la sociedad.

Este cambio se ha producido en gran medida por la irrupción de internet y las herramientas de la web 2.0, que han facilitado como ningún otro medio la comunicación multidireccional (Weitze, 2017), construyendo un espacio conversacional (Grand et al., 2016; Brown, 2016) que estimula una ciudadanía más activa (Coleman, 2001).

Los trabajos que estudian el impacto de internet y los instrumentos digitales en la comunicación pública de la ciencia son todavía exiguos y la bibliografía científica sobre el uso y la eficacia de este nuevo canal para implicar a los ciudadanos en el proceso científico es casi inexistente. Esto se debe a la juventud del modelo de participación del público en la ciencia, así como a la propia naturaleza cambiante de la Red.

Este trabajo pretende contribuir a generar una línea académica en este sentido. Para ello se ha analizado el uso que los proyectos de investigación españoles financiados por el European Research Council (ERC) hacen de los medios digitales para implicar a la sociedad en el proceso científico, así como las características y efectividad de ese uso. La muestra está constituida por los proyectos españoles que consiguieron financiación del ERC en 2015.

Seguidamente se presenta el estado de la cuestión, así como la metodología aplicada, que se sustenta en un cuestionario diseñado ad hoc, elaborado y validado mediante el método Delphi. A continuación se detallan los resultados obtenidos, que apuntan al escaso uso de las herramientas digitales y a la existencia de una comunicación unidireccional y dirigida fundamentalmente a expertos, y las conclusiones que de éstos se derivan.

\section{Modelo de participación del público en la ciencia desde la dimensión de la investigación e innovación responsables}

Para comprender el modelo de participación del público en la ciencia desde la dimensión de la investigación e innovación responsables (Responsible Research and Innovation, RRI) es necesario contextualizar las implicaciones de este sistema de I+D+i. Aunque la bibliografía científica ha planteado múltiples aproximaciones a este concepto, la que propone la Comisión Europea (2014) sintetiza los aspectos anteriormente propuestos.

Desde esta perspectiva la investigación e innovación responsable es aquella en la que los actores sociales trabajan juntos durante el curso de la investigación con el objetivo de alinear los procesos y sus resultados con los valores, necesidades y expectativas de la sociedad. Se trata de un término amplio e integrado por seis dimensiones: participación del público en la ciencia, acceso abierto, igualdad de género, educación científica, ética y gobernanza (Comisión Europea, 2015).

Académicos de la comunicación pública de la ciencia han contribuido a definir el modelo de la participación del público en la ciencia desde diferentes perspectivas. Rowe y Frewer (2005) consideran la implicación del público en el proceso científico como una combinación entre la comunicación, la consulta y la participación del público en el marco de la investigación y la innovación. Por su parte, Ravn y Mejlgaard (2014) parten de la categorización de Rowe y Frewer (2005) y plantean una clasificación que engloba las iniciativas de participación del público como: comunicación pública, activismo público, consulta pública y deliberación pública.

Otros académicos como Bonney et al. (2009) definen la participación del público ciñéndola a las etapas del proceso de investigación e innovación en las que los ciudadanos pueden ser partícipes.

Este concepto también se ha asociado con la implicación de los investigadores en la comunicación de los resultados científicos (Bauer; Jensen, 2011). Conferencias públicas, entrevistas en medios de comunicación, redacción de libros de 
divulgación, participación en debates públicos, o colaboración con organizaciones no gubernamentales, son algunas de las actividades integradas en esta definición.

\section{Internet como canal de interacción entre ciencia y sociedad}

El desarrollo del modelo de participación pública de la ciencia ha transcurrido de forma paralela a la consolidación de internet como uno de los principales canales de comunicación de la sociedad contemporánea (Acord; Harley, 2013; Castells, 2001; 2011; Middaugh; Kahne, 2013; Papacharissi, 2002). En la propia naturaleza de la web 2.0 están implícitos los conceptos de interacción y conversación (Grand et al., 2016; Brown, 2016; Könneker; Lugger, 2013; Weilgod; Treise, 2004; Flores-Vivar, 2009; Castells, 2001) indispensables en la implementación de investigación e innovación responsable.

Las ventajas evidenciadas del entorno digital son coincidentes en la bibliografía científica publicada. Varios autores han establecido como principales beneficios de la web 2.0 en la comunicación pública de la ciencia:

- creación de un espacio conversacional (Grand et al., 2016; Brown, 2016; Weilgod; Treise, 2004);

- visibilización de la producción científica (López-Goñi; Sánchez-Agudo, 2018; Wolf, 2017; Shuai; Pepe; Bolen, 2012);

- fomento de debate y discusión sobre controversias científicas (Ke; Ahn; Sugimoto, 2017);

- transformación de la comunicación unidireccional a la bidireccional (Weitze, 2017; Brossard; Schefeule, 2013; Weilgod; Treise, 2004).

El interés académico en la dimensión digital de la comunicación pública de la ciencia como objeto de estudio es incipiente y aún es más exiguo en lo que respecta a la participación del público en la ciencia. Los trabajos académicos que han abordado la evaluación de la implicación del público en la ciencia se han centrado en la faceta convencional a través del diseño de sistemas de evaluación (Comisión Europea, 2015; 2018; Meijer et al., 2016) que no integran la esfera digital como objeto de análisis. Sólo un número limitado de estudios (López-Pérez; Olvera-Lobo, 2018a; 2018b; Neresini; Bucchi, 2011) han abordado la validación científica de indicadores que permitan estudiar el papel de las redes sociales y de la Web en la participación del público en la ciencia.

Según lo expuesto, a pesar de las cualidades de internet como canal para favorecer la participación ciudadana en la ciencia, no hay resultados empíricos que demuestren si este nuevo canal ha reducido la brecha entre científicos y sociedad, una pregunta de investigación a cuya respuesta pretende contribuir el presente trabajo.

\section{Metodología}

Con el fin de obtener criterios e indicadores validados consensuadamente para analizar la implicación digital del público en la ciencia de excelencia, se ha aplicado el método Delphi (Osborne et al., 2003; Clayton, 1997; Murry; Hammons, 1995). Éste ha sido utilizado anteriormente para el diseño de propuestas metodológicas de análisis de la comunicación de la ciencia (Ouarichi; Olvera-Lobo; Pérez-Gutiérrez, 2017; Seakins; Dillon, 2013) y de la educación científica (Smith; Simpson, 1995; Blair; Uhl, 1993). Se trata de un proceso sistemático, interactivo y grupal encaminado a la obtención de opiniones y consenso, a partir de las experiencias y juicios subjetivos de un grupo de expertos (Scapolo; Miles, 2006; Osborne et al., 2003).

Para la aplicación del Delphi en este trabajo, la selección de expertos se ha realizado teniendo en cuenta cuatro aspectos:

- área de conocimiento afín a la materia abordada que los acredita para validar el cuestionario;

- relevancia en el área;

- igualdad de género;

- diversidad geográfica.

De este modo, las áreas de conocimiento han sido:

- comunicación pública de la ciencia;

- educación científica;

- investigación e innovación responsables;

- científicos blogueros;

- redes sociales.

Los criterios para medir la relevancia en el área han sido:

- calidad y cantidad de publicaciones científicas;

- experiencia profesional y académica en el área;

- impacto social (este ítem se ha fijado principalmente en el caso de los científicos blogueros y es relativo al número de seguidores);

- formación;

- coordinación y organización de proyectos internacionales que implican la participación del público en el proceso de investigación o que están vinculados con la investigación e innovación responsables.

También se han seguido valores como la igualdad de género y de los 14 participantes, 8 fueron mujeres y 6 hombres. 
Asimismo, se consideró relevante la procedencia geográfica haciendo especial hincapié en aquellos países, además de España, en los que se están realizando importantes esfuerzos para la implementación de la RRI, como Reino Unido, Alemania y Dinamarca, y que por tanto poseen un mayor conocimiento de las ventajas y desventajas de este modelo.

Tabla 1. Expertos método Delphi

\begin{tabular}{|c|c|c|}
\hline Área & Experto & Origen \\
\hline \multirow{3}{*}{ Comunicación pública de la ciencia } & Profesor de comunicación científica & Reino Unido \\
\hline & Técnica en participación pública de la ciencia & Reino Unido \\
\hline & Periodista científico & España \\
\hline \multirow{3}{*}{ Educación científica } & Profesora de educación científica & Alemania \\
\hline & Educadora científica en un museo de ciencia & Alemania \\
\hline & Educadora científica en un museo de ciencia & España \\
\hline \multirow{2}{*}{ Investigación e innovación responsables (RRI) } & Experta en gestión de proyectos RRI & España \\
\hline & Experta en humanidades digitales y RRI & España \\
\hline \multirow{2}{*}{ Blogueros especializados en ciencia } & Bloguero especializado en ciencia & España \\
\hline & Bloguero especializado en ciencia & España \\
\hline \multirow{4}{*}{$\begin{array}{l}\text { Comunicación pública de la ciencia en redes } \\
\text { sociales }\end{array}$} & Profesora de medios interactivos & Dinamarca \\
\hline & Profesor de comunicación en social media & España \\
\hline & Experto en comunicación pública de la ciencia a través de los social media & España \\
\hline & Experta en comunicación pública de la ciencia a través de los social media & España \\
\hline
\end{tabular}

El aspecto clave en el desarrollo de la metodología de trabajo ha sido conseguir el consenso del grupo, pero con la máxima autonomía por parte de los participantes. Para ello se han realizado tres rondas de consultas en un proceso interactivo y anónimo que ha permitido a los participantes opinar, recibir las conclusiones del resto del grupo en cada una de las rondas y, finalmente, reconsiderar sus opiniones en una última fase. Los criterios e indicadores de evaluación establecidos por consenso son los que se muestran en la tabla 2.

Tabla 2. Criterios de evaluación validados por el método Delphi

\begin{tabular}{|c|c|}
\hline Criterios & Indicadores \\
\hline \multirow{5}{*}{ Identificación } & Nombre del proyecto de investigación \\
\hline & Institución responsable \\
\hline & Fecha de comienzo y cierre del proyecto \\
\hline & Transdisciplinariedad \\
\hline & Países implicados en el proyecto \\
\hline \multirow{4}{*}{$\begin{array}{l}\text { Tipos de herramientas } \\
\text { online }\end{array}$} & Webs. Existencia de una web específica para los proyectos de investigación \\
\hline & Redes sociales (Facebook, Twitter, YouTube e Instagram) \\
\hline & Blogs. Uso de blogs para la comunicación de los resultados científicos o para promover la participación ciudadana \\
\hline & Apps. Uso de aplicaciones para recopilar información u opiniones del público \\
\hline \multirow{4}{*}{$\begin{array}{l}\text { Categoría de } \\
\text { participación }\end{array}$} & Comunicación \\
\hline & Consulta \\
\hline & Participación \\
\hline & Co-creación \\
\hline \multirow{3}{*}{$\begin{array}{l}\text { Mecanismos de } \\
\text { participación }\end{array}$} & Encuestas \\
\hline & Materiales educativos \\
\hline & Consultas. Recopilar opiniones a favor o en contra en alguna de las fases del proceso de investigación \\
\hline \multirow{12}{*}{$\begin{array}{l}\text { Características de la } \\
\text { participación }\end{array}$} & Facilidad de acceso a la web y perfiles sociales \\
\hline & Nivel de comunicación (uni o bidireccional) \\
\hline & Dirección de la comunicación (científico-público/público-científico/científico-científico/público-público) \\
\hline & Lenguaje utilizado en la comunicación \\
\hline & Tipo de público \\
\hline & Expertos implicados \\
\hline & Presentación de suficiente información \\
\hline & Comunicación de los resultados de la participación \\
\hline & Dirección de correo electrónico para contacto del público \\
\hline & Tipo de información presentada al público \\
\hline & Acceso abierto \\
\hline & Tema de discusión \\
\hline \multirow{4}{*}{$\begin{array}{l}\text { Intensidad de la } \\
\text { participación }\end{array}$} & Número de seguidores en redes sociales \\
\hline & Número de personas implicadas en cada categoría de la participación \\
\hline & Cuantificación de la participación \\
\hline & Demanda de la información por parte del público \\
\hline
\end{tabular}


En la primera ronda los expertos evaluaron en una escala Likert ( 1 = importancia baja, 2 = importancia media, o $3=$ importancia alta) un conjunto de criterios e indicadores basados en las definiciones y aproximaciones al concepto de participación del público en la ciencia planteadas por la bibliografía científica, y se añadieron tres preguntas abiertas que perseguían:

I) evaluar la idoneidad del objeto de estudio -¿considera que internet y las herramientas online son un buen canal para promover la participación del público en el desarrollo científico y tecnológico?-, o

II) mejorar y ampliar los criterios e indicadores propuestos por el grupo coordinador -¿qué criterios y/o indicadores añadiría para la mejora de un sistema de evaluación de la participación del público a través de herramientas online?, ¿qué criterios y/o indicadores eliminaría para la mejora de un sistema de participación del público a través de las herramientas online?-.

Con el fin de incluir las aportaciones de los expertos a través de las preguntas abiertas y someterlas al consenso del grupo, el cuestionario enviado en la segunda ronda incluyó los indicadores propuestos e integró aquellos que en la primera ronda no habían alcanzado una valoración promedio de 2 puntos sobre 3, que fue lo establecido como consenso para su inclusión. El objetivo de incluir estos indicadores fue someter a una segunda reflexión la consideración de los mismos, tal y como marca el método Delphi, antes de eliminarlos definitivamente del cuestionario.

A partir de las respuestas obtenidas, se diseñó la tercera versión del cuestionario, en el que se incluyeron los indicadores que habían logrado consenso en la primera y segunda ronda. En este caso el objetivo se centró en comprobar la estabilidad en las respuestas entre los cuestionarios 1, 2 y 3, e integrar de forma definitiva aquellos indicadores que hubiesen alcanzado un consenso superior a una valoración promedio de 2 puntos.

El proceso, que se ha descrito en trabajos anteriores (López-Pérez; Olvera-Lobo, 2018a; 2018b) concluyó al alcanzarse el criterio de saturación establecido por el consenso y la estabilidad de las valoraciones de los expertos de los indicadores incluidos en el cuestionario.

\section{Muestra de estudio}

La muestra de estudio está constituida por los proyectos financiados por el European Research Council a través de sus convocatorias Starting grant; Consolidator grant; Advanced grant; Proof of concept y Synergy grant. En las cuatro primeras se han seleccionado los proyectos españoles que recibieron financiación en 2015 y en el caso de las Synergy grants se han integrado los beneficiarios de 2013 por ser la última convocatoria publicada de este programa. Suman un total de 67 proyectos.

La elección de los proyectos financiados por el $E R C$ responde a tres criterios:

- excelencia: el organismo europeo se rige por este valor para destinar sus recursos;

- temporalidad: se han seleccionado los financiados en 2015 para no sesgar los resultados de participación y comunicación en proyectos que hayan sido financiados recientemente y se encuentren en su fase inicial. En los de 2015 las investigaciones se encuentran en una fase intermedia que ya ha permitido el desarrollo de estrategias de difusión e implicación del público en el proceso científico. La recogida de datos se llevó a cabo durante el mes de abril de 2018;

- coherencia: se refiere al hecho de que la Unión Europea ha sido la impulsora de la implementación de la investigación e innovación responsables (RRI) dentro del programa Horizon 2020 y, por coherencia, los proyectos que reciben su financiación deberían responder a los valores de la RRI, entre los que se integra la participación del público en el proceso de investigación.

\section{Resultados}

Los resultados se presentan según los diferentes criterios considerados:

\subsection{Identificación}

El hecho de que sólo un 23,9\% de los proyectos de investigación analizados tenga web ha propiciado que el análisis del criterio de identificación se extienda más allá de los medios de comunicación específicamente diseñados para la difusión de los proyectos. La información más detallada de los proyectos aparece publicada en la web del ERC, en la que en el $100 \%$ de los casos se publica el nombre en inglés del proyecto y se identifica la entidad beneficiaria, así como el investigador principal.

Las fechas de inicio y conclusión de los proyectos también se detallan en la web del ERC. El 76,1\% de los proyectos comenzó en 2016, un $13,7 \%$ en 2017, un 11,5\% en 2015 y un 5,8\% en 2014 . En cuanto a su conclusión, un $28,2 \%$ ha finalizado antes de 2018, un 4,4\% lo hará entre 2019 y 2020 y un 67,4\% en 2021 . El hecho de que la mayoría de los proyectos esté en el estadio intermedio de ejecución incita a la repetición del análisis a su conclusión para determinar la evolución del uso de medios de participación del público en las distintas fases de los procesos de investigación.

La transdisciplinariedad se reseña en el $19,4 \%$ de los casos. Por último, el indicador relativo a países implicados, en todos los proyectos se indica únicamente el país de la institución beneficiaria, en este caso España. 


\subsection{Tipos de medios online}

Los proyectos de investigación analizados hacen un escaso uso de los sistemas online en las dos dimensiones de la participación pública en la ciencia, es decir, tanto en lo que se refiere a la comunicación como en lo relacionado con la interacción-implicación de los ciudadanos. Esto es algo que se refleja en los reducidos porcentajes obtenidos del uso de los tipos de herramientas. Como muestra el gráfico 1 , un 23,9\% de los proyectos analizados cuentan con una web, el medio más usado, seguido de Twitter: un 13,4\% tiene cuenta en esta red de microblogging. El uso de YouTube, Facebook y de blogs es aún más escaso con un $9 \%$, un $7,5 \%$ y un $6 \%$ respectivamente. Las herramientas más minoritarias son Instagram y otras apps, que comparten un 1,5\%.

Los resultados evidencian además una preponderancia de los canales de comunicación unidireccionales sobre los que permiten interacción y diálogo. Este escenario sugiere que el papel del público en la dimensión digital de la ciencia sigue siendo de usuario final y no de participante activo.

\subsection{Categoría de participación}

Los proyectos analizados no fomentan el uso de internet y de las herramientas web 2.0 como canales de interacción con la sociedad. Un 12,1\% de los proyectos emplea estrategias de comunicación unidireccional (difusión de información sin diálogo o interacción) y, dentro de éstos, solo el $9 \%$ cuenta con un canal de noticias para divulgar sus principales resultados.

En cuanto a la implicación activa del público en el proceso de investigación, en las categorías de consulta y cocreación esta implicación es inexistente. Sólo un 3\% de los proyectos analizados emplea alguna de las herramientas digitales analizadas para hacer posible la participación de los ciudadanos en la recogida de datos para el proyecto o a través de la recopilación de ideas sobre el mismo.

\subsection{Mecanismos de participación}

Un $9 \%$ de los proyectos utiliza materiales educativos y un 3\% mecanismos de consulta. El resto de items contemplados en el cuestionario de evaluación no son utilizados en ningún caso. Los recursos educativos más utilizados son la publicación de conferencias, documentales o vídeos online para visibilizar sus logros y líneas de investigación.

\subsection{Características de la participación}

El fácil acceso a su web o a los perfiles sociales creados para el proyecto indudablemente facilitan su visibilización y difusión. La facilidad de acceso se ha evaluado con una puntuación de 0 a 5, siendo 0 muy difícil y 5 muy fácil. Como se observa en el gráfico 2, el estudio muestra que el $53,7 \%$ de las páginas web son de muy difícil acceso, es decir, no están ubicadas en sitios visibles como las páginas principales de las instituciones beneficiarias, el $19,4 \%$ son de difícil acceso y tan sólo un $26,9 \%$ están publicitadas en espacios fácilmente accesibles (gráfico 2). En cuanto a los perfiles sociales, en el $82,8 \%$ de los casos están publicados en espacios no visibles o de difícil acceso.

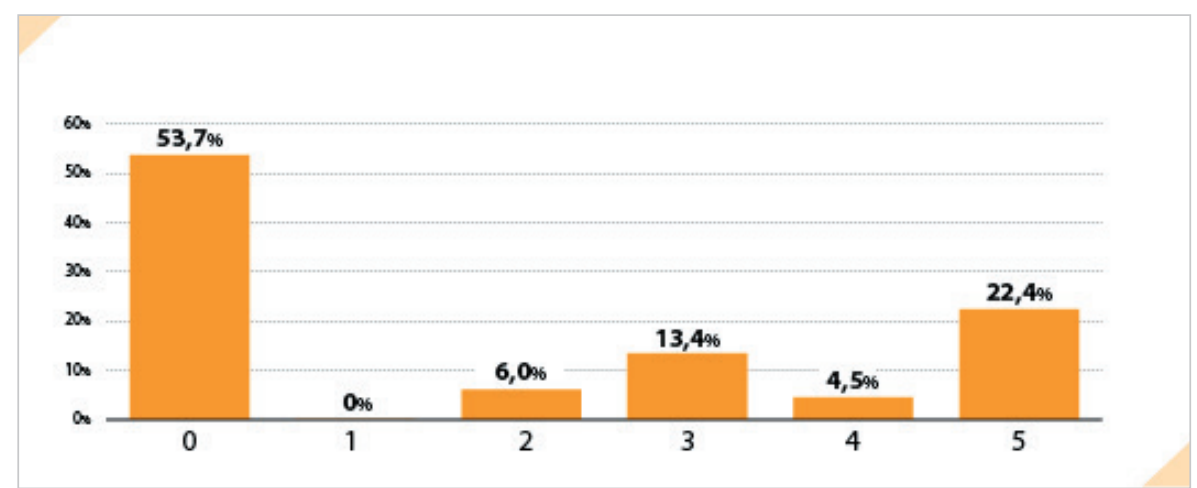

Gráfico 2. Facilidad de acceso a la web

Aunque la web 2.0 se caracteriza por ser un espacio conversacional que facilita el diálogo y la interacción, el $97 \%$ de la comunicación que se realiza en las redes sociales analizadas es unidireccional, y se produce de arriba abajo, es decir desde la institución-responsable del proyecto hacia la sociedad.

El lenguaje utilizado en las comunicaciones es técnico en el $80,6 \%$ de los casos, lo que probablemente no contribuye a potenciar el acercamiento al ciudadano medio. Esta especialización en el contenido hace que la dirección de la comunicación sea en un $83,6 \%$ de los casos de científico a científico y el 16,4\% científico-público (gráfico 3). En cuanto al tipo de 
público no es posible definirlo al no existir comentarios de usuarios en las redes analizadas que nos permitan inferir sus características.

El 93,8\% de los proyectos que sí utilizan las herramientas de la web 2.0 para interaccionar con el público, realiza una comunicación institucional frente a la personal elegida por un 6,2\% de los proyectos (gráfico 4).

En lo que respecta a la información sobre el proyecto como sus objetivos o líneas de investigación, el 76,1\% publica una información insuficiente (entendida la información del proyecto como aquella que incluye el título, objetivos, metodología, período de ejecución, líneas de investigación a desarrollar y presupuesto) frente al $23,9 \%$ que sí ofrece una información completa.

La publicación de un correo electrónico de contacto se da en un $22,4 \%$ de los casos, frente al $77,6 \%$ que no integra esta información.

Con respecto a la publicación de resultados científicos en abierto, tal y como puede observarse en el gráfico 4 , el $40,3 \%$ de los proyectos con website publica en abierto sus resultados, frente al $59,7 \%$ que no lo hace (gráfico 4).

Finalmente, en referencia a la información sobre los proyectos que los beneficiarios publican online, en el $65,6 \%$ de los casos se publica un resumen del proyecto. El $57,1 \%$ de los proyectos se publican en la web del grupo de investigación, el 40,5\% en la sección de investigación de la web de la institución beneficiaria de la financiación del $E R C$ y sólo en un 2,4\% la información es publicada en los portales de transparencia de estas mismas instituciones (gráfico 5).

\subsection{Intensidad de la participación}

La red social que presenta mayor intensidad de participación en términos de seguidores y posts publicados es Twitter: el $9,1 \%$ de perfiles supera los 5.000 seguidores, otro $9,1 \%$ tiene entre 1.000 y 5.000 seguidores y el $81,8 \%$ restante tiene menos de mil. En el caso de Facebook las cifras son similares, el $80 \%$ cuenta con menos de 1.000 seguidores mientras que el $20 \%$ tiene más de 5.000 y ninguno entre 1.000 y 5.000 .

En lo que respecta a la publicación y actualización de contenidos, vuelve a ser Twitter la red con mayor actividad: un 22,2\% de los proyectos con perfil en Twitter publicó más de 50 tweets en un mes mientras que en el 77,8\% de los casos 
no se superan los 20 tweets mensuales (no hay perfiles con publicaciones entre 21 y 49 tweets). En el caso de Facebook el $50 \%$ de los proyectos publicó menos de 10 posts y el otro $50 \%$ entre 10 y 24 . En YouTube, el $60 \%$ publicó 3 vídeos en el período analizado, un $20 \% 1$ vídeo y el $20 \%$ restante ninguno. El único proyecto con presencia en Instagram no publicó ningún post en el período analizado.

\section{Conclusiones}

La ciencia de excelencia española aún no ha encontrado en internet un canal útil de interacción con los ciudadanos. Esto es lo que sugieren los resultados obtenidos sobre el uso de la web 2.0. Sólo un 23,9\% cuenta con una web, y menos de un $15 \%$ ha creado perfiles en redes como Facebook, Twitter o YouTube. Además, cuando tienen un perfil la repercusión de las informaciones publicadas en el mismo tienen muy poco impacto por el escaso número de seguidores, y es que el $80 \%$ de los perfiles tiene menos de 5.000 usuarios. Por otro lado destaca la escasa publicación de contenidos en estas redes -el $50 \%$ de los proyectos publica menos de 10 posts mensuales-.

Un aspecto que podría explicar el reducido número de seguidores es el difícil acceso tanto a sus páginas web como a los perfiles sociales. Es destacable el hecho de que sólo el 40,5\% de las instituciones beneficiarias publique información del proyecto y de su financiación en sus páginas principales, y que un reducido $2,4 \%$ integre esta información en sus portales de transparencia. La información más completa se publicó en más de un $50 \%$ de los casos en las páginas propias de los grupos de investigación, un resultado que confirma las conclusiones ya apuntadas por Pacios, Vianello-Osti y Rodríguez-Bravo (2016) en un trabajo sobre transparencia y acceso a la información de los proyectos de investigación en las universidades públicas españolas.

Pese a que entre las principales características de la web 2.0 destacan la interactividad, el diálogo y la participación, sólo el $3 \%$ de los proyectos utiliza los canales digitales con este objetivo, y en un $77,6 \%$ de las webs no se incluye siquiera una dirección de correo electrónico para contactar con los miembros del equipo investigador. Esta circunstancia demuestra que la implementación de la dimensión digital de la participación pública dentro la investigación e innovación responsable en España está aún en una fase incipiente y que no está asumida por los investigadores. El 97\% de la comunicación que se realiza en las redes sociales analizadas es unidireccional, y se produce de arriba abajo, es decir desde la institución-responsable del proyecto a la sociedad.

Otra dimensión de la RRI que aún no está consolidada, según los resultados obtenidos, es el acceso abierto a la investigación y sus resultados. El $60 \%$ de los proyectos no publica información en abierto sobre sus resultados. Aunque este dato habría que contrastarlo una vez finalicen los proyectos (el 67,4\% concluirá en 2021) ya que en muchos de ellos los resultados relevantes posiblemente se obtengan en la última fase del proceso.

Según se observa, en el entorno digital los equipos de investigación están más preocupados por la difusión de la ciencia entre científicos que por la comunicación con el público. En más del $80 \%$ de los casos utilizan lenguaje técnico y dirigen sus mensajes a los expertos. La comunicación es fundamentalmente institucional y hay escasa personalización de los mensajes, a pesar de que existen evidencias científicas de que en el caso de los medios sociales la autenticidad y la conexión personal se han establecido como criterios adicionales para el fomento de la credibilidad de la información (Betsch et al., 2010).

Por otro lado, el análisis realizado destaca la importancia marginal que la participación del público tiene en la ciencia de excelencia en España y la necesidad de trabajar en este sentido, tanto para su implementación como para su evaluación. Por esto, aunque este estudio se ha concentrado principalmente en determinar los esfuerzos que lleva a cabo la ciencia excelente por involucrar a los ciudadanos más que en el estudio de la calidad de esos esfuerzos, los indicadores validados pueden contribuir al desarrollo de análisis centrados en evaluar la efectividad de la participación digital del público en la ciencia en nuestro país.

\section{Referencias}

Acord, Sophia-Krzys; Harley, Diane (2013). "Credit, time and personality: The human challenges to sharing scholarly". New media \& society, v. 15, n. 3, pp. 379-397. https://doi.org/10.1177/1461444812465140

Bauer, Martin W.; Jensen, Pablo (2011). "The mobilization of scientists for public engagement". Public understanding of science, v. 20, n. 1, pp. 3-11 https://doi.org/10.1177/0963662510394457

Betsch, Cornelia; Renkewitz, Frank; Betsch, Tilmann; Ulshöfer, Corina (2010). "The influence of vaccine-critical websites on perceiving vaccination risks". Journal of health psicology, n. 15, v. 3, pp. 446-455.

https://doi.org/10.1177/1359105309353647

Blair, Shirley; Uhl, Norman P. (1993). "Using the Delphi method to improve the curriculum". The Canadian journal of higher education, v. 23, n. 3, pp. 107-128. 
http://journals.sfu.ca/cjhe/index.php/cjhe/article/view/183175

Bonney, Rick; Ballard, Heidi; Jordan, Rebecca; McCallie, Ellen; Phillips, Tina; Shirk, Jennifer; Wilderman, Candie C. (2009). Public participation in scientific research: Defining the field and assessing its potential for informal science education. A Caise Inquiry Group Report. Washington, D.C., EUA: Center for Advancement of Informal Science Education (Caise). http://www.birds.cornell.edu/citscitoolkit/publications/CAISE-PPSR-report-2009.pdf

Brossard, Dominique; Scheufele, Dietram (2013). "Science, new media, and the public". Science, v. 339, n. 6115, pp. 40-41.

https://doi.org/10.1126/science.1232329

Brown, David J. (2016). Access to scientific research. Challenges facing communications in STM. Berlin: Walter de Gruyter. ISBN: 9783110376169

https://doi.org/10.1002/asi.23764

Castells, Manuel (2001). La galaxia de internet. Barcelona: Areté. ISBN: 9788401386060

Castells, Manuel (2011). Comunicación y poder. Madrid: Alianza Editorial. ISBN: 9788420684994

Clayton, Mark (1997). "Delphi: a technique to harness expert opinion for critical decision-making tasks in education". Educational psychology, n. 17, v. 4, pp. 373-386.

https://doi.org/10.1080/0144341970170401

Coleman, Stephen (2001). "The transformation of citizenship?" In: Axford, Barrie; Huggins, Richard (eds.). New media and politics. London: SAGE, pp. 109-126. ISBN: 9780761962007

https://doi.org/10.4135/9781446218846.n5

Comisión Europea (2014). Responsible research and innovation - Europe's ability to respond to societal challenges. https://ec.europa.eu/research/swafs/pdf/pub_rri/KIO214595ENC.pdf

Comisión Europea (2015). Indicators for promoting and monitoring responsible research and innovation. http://ec.europa.eu/research/swafs/pdf/pub_rri/rri_indicators_final_version.pdf

Comisión Europea (2018). Monitoring the evolution and benefits of responsible research and innovation. https://www.rri-tools.eu/-/morri-monitoring-the-evolution-and-benefits-of-responsible-research-and-innovation

Flores-Vivar, Jesús-Miguel (2009). “Nuevos modelos de comunicación, perfiles y tendencias en las redes sociales”. Comunicar, v. 17, n. 33, pp. 73-81.

https://doi.org/10.3916/c33-2009-02-007

Grand, Ann; Holliman, Richard; Collins, Trevor; Adams, Anne (2016). "'We muddle our way through': Shared and distributed expertise in digital engagement with research". JCOM, v. 15, n. 4, A05.

https://doi.org/10.22323/2.15040205

Ke, Quing; Ahn, Yong-Yeol; Sugimoto, Cassidy R. (2017). "A systematic identification and analysis of scientists on Twitter". PLoS one, v. 12, n. 4.

https://doi.org/10.1371/journal.pone.0175368

Könneker, Carsten; Lugger, Beatrice (2013). “Public science 2.0 - back to the future”. Science, v. 342, n. 6154, pp. 49-50. https://doi.org/10.1126/science.1245848

López-Goñi, Ignacio; Sánchez-Angulo, Manuel (2018). "Social networks as a tool for science communication and public engagement: focus on Twitter". FEMS Microbiology letters, v. 365, n. 2.

https://doi.org/10.1093/femsle/fnx246

López-Pérez, Lourdes; Olvera-Lobo, María-Dolores (2018a). “Public engagement in science via web 2.0 technologies. Evaluation criteria validated using the Delphi method". JCOM, v. 17, n. 2.

https://doi.org/10.22323/2.17020208

López-Pérez, Lourdes; Olvera-Lobo, María-Dolores (2018b). “Criterios para la evaluación de la implicación del público en la ciencia a través de la web 2.0". En: Romero-Frías, Esteban; Bocanegra-Barbecho, Lidia (eds.). Ciencias sociales y humanidades digitales aplicadas. Casos de estudio y perspectivas críticas. Granada: Universidad de Granada; Downhill Publishing (NY), 2018. ISBN: 9788433863188

http://digibug.ugr.es/handle/10481/54383

Marschalek, Ilse (2017). Public engagement in responsible research and innovation. A critical reflection from the practitioner's point of view. Wien: University of Wien.

https://www.zsi.at/object/publication/4498/attach/Marschalek_Public_Engagement_in_RRI.pdf

Meijer, Ingeborg; Mejlgaard, Niels; Woolley, Richard; Rafols, Ismael; Wroblewski, Angela (2016). "Monitoring the evolution and benefits of responsible research and innovation (MoRRI) - a preliminary framework for RRI dimensions \& 
indicators". In: $21^{\text {st }}$ Int/ conf on science and technology indicators: Peripheries, frontiers and beyond. http://hdl.handle.net/10261/161892

Middaugh, Ellen; Kahne, Joseph (2013). “Nuevos medios como herramienta para el aprendizaje cívico”. Comunicar, v. 20, n. 40, pp. 99-108.

https://doi.org/10.3916/c40-2013-02-10

Murry, John W.; Hammons, James O. (1995). "Delphi: A versatile methodology for conducting qualitative research". The review of higher education, v. 18, n. 4, pp. 423-436.

https://doi.org/10.1353/rhe.1995.0008

Neresini, Federico; Bucchi, Massimiano (2011). "Which indicators for the new public engagement activities? An exploratory study of European research institutions". Public understanding of science, v. 20, n. 1, pp 64-79.

https://doi.org/10.1177/0963662510388363

Osborne, Jonathan; Collins, Sue; Ratcliffe, Mary; Milar, Robin; Duschl, Rick (2003). "What 'ideas-about-science' should be taught in school science? A Delphi study of the expert community". Journal of research in science teaching, v. 40, n. 7, pp. 692-720.

https://doi.org/10.1002/tea.10105

Ouariachi, Tania; Olvera-Lobo, María-Dolores; Gutiérrez-Pérez, José (2017). “Analyzing climate change communication through online games". Science communication, v. 39, n. 1, pp. 10-44

https://doi.org/10.1177/1075547016687998

Pacios, Ana-Reyes; Vianello-Osti, Marina; Rodríguez-Bravo, Blanca (2016). "Transparencia y acceso a la información sobre proyectos de investigación en las universidades públicas españolas". El profesional de la información, v. 25, n. 5, pp. 721-729.

https://doi.org/10.3145/epi.2016.sep.02

Papacharisi, Zizi (2002). "The virtual sphere: The internet as a public sphere”. New media \& society, n. 4, v. 1, pp. 9-27. https://doi.org/10.1177/14614440222226244

Ravn, Tine; Mejlgaard, Niels (eds.) (2014). Public engagement innovation for Horizon 2020. Catalogue of PE initiatives. University of Helsinky; University of Aarhus; Vilnius University International Business School; Laboratorio di Scienze della Cittadinanza.

http://www.vm.vu.It/uploads/pdf/Public_Engagement_Innovations_H2020-2.pdf

Rowe, Gene; Frewer, Lynn J. (2005). “A typology of public engagement mechanisms". Science, technology \& human values, v. 30, n. 2, pp. 251-290.

https://doi.org/10.1177/0162243904271724

Scapolo, Fabiana; Miles, Ian (2006). "Eliciting experts' knowledge: A comparison of two methods". Technological forecasting and social change, n. 73, v. 6 pp. 679-704.

https://doi.org/10.1016/j.techfore.2006.03.001

Seakins, Amy; Dillon, Justin (2013). "Exploring research themes in public engagement within a Natural History Museum: A modified Delphi approach". International journal of science education, v. 3, n. 1, pp. 52-76.

https://doi.org/10.1080/21548455.2012.753168

Shuai, Xin; Pepe, Alberto; Bolen, Johan (2012). "How the scientific community reacts to newly submitted preprints: Article downloads, Twitter mentions, and citation". PLoS one, v. 7, n. 11.

https://doi.org/10.1371/journal.pone.0047523

Smith, Kathleen S.; Simpson, Ronald D. (1995). "Validating teaching competencies for faculty members in higher education: A national study using the Delphi method". Innovative higher education, v. 19, n. 3, pp. 223-234.

https://doi.org/10.1007/bf01191221

Weigold, Michael F.; Treise, Debbie (2004). "Attracting teen surfers to science web sites". Public understanding of science, v. 13, n. 3, pp. 229-248.

https://doi.org/10.1177/0963662504045504

Weitze, Marc-Denis (2017). Social media and digital science communication. Analysis and recommendations for dealing with risks and opportunities in a democracy. Berlin: National Academy of Science and Engineering, German National Academy of Sciences Leopoldina and Union of the German Academies of Sciences and Humanities. ISBN: 9783804736320 https://www.acatech.de/wp-content/uploads/2018/03/WOM2_EN_web_final.pdf

Wolf, Julie M. (2017). "The multipurpose tool of social media applications for scientists, science communicators and educators". Clinical microbiology newsletter, v. 39, n. 10, pp. 75-79.

https://doi.org/10.1016/j.clinmicnews.2017.04.003 